\title{
ESTUDIO COMPARATIVO DE LAS COMUNIDADES DE MILPIÉS (ARTHROPODA: DIPLOPODA) PRESENTES EN LA SABANA DE PAJÓN, EL PINAR Y EL BOSQUE LATIFOLIADO DEL PARQUE NACIONAL VALLE NUEVO, REPÚBLICA DOMINICANA
}

\author{
Katihusca Rodríguez Soto y Carlos Suriel
}

Museo Nacional de Historia Natural "Prof. Eugenio de Jesús Marcano". Calle César Nicolás Penson, Plaza de la Cultura Juan Pablo Duarte, Santo Domingo, República Dominicana. k.rodriguez@mnhn.gov.do,c.suriel@mnhn.gov.do

\section{RESUMEN}

Se comparó la diversidad de las comunidades de diplópodos en áreas delimitadas de tres tipos de vegetación del Parque Nacional Valle Nuevo (sabana de pajón, pinar y bosque latifoliado) durante la temporada lluviosa. Se establecieron tres estaciones de estudio correspondientes a los tres tipos de vegetación considerados. La riqueza total registrada fue 15 especies. El bosque latifoliado fue el tipo de vegetación que registró el más alto valor en riqueza de especies (12), mientras que la sabana de pajón fue el que presentó el valor más bajo con ocho especies. La mayor abundancia de especímenes la registró el pinar (205) y la menor la presentó el bosque latifoliado (73). Las especies más abundantes del estudio fueron Achromoporus platyurus y Prostemmiulus setosus. La comunidad del bosque latifoliado fue la más diversa de acuerdo con el valor obtenido con el índice de diversidad de Shannon y el recíproco del índice de dominancia de Simpson $\left(H^{\prime}=2.25 ; \mathrm{D}=8.91\right)$. La sabana de pajón mostró ser la más pobre en relación con la diversidad alfa según los resultados de estos índices $\left(\mathrm{H}^{\prime}=1.42 ; \mathrm{D}=3.32\right)$. El índice de similitud de Jaccard arrojó el más bajo valor al relacionar los ecosistemas sabana de pajón y bosque latifoliado (50\%), mientras que la mayor similitud se observó al comparar la sabana de pajón con el pinar (72\%). Este último resultado fue estadísticamente significativo para una probabilidad (p) de 0.05 , mostrando que existe un alto grado de semejanza faunística en cuanto a los diplópodos entre ambos tipos de vegetación. Esta alta similitud podría deberse a la homogeneidad estructural de estos dos tipos de vegetación. Se hizo un dendrograma que reflejó estos mismos resultados. Las curvas de acumulación de especies y estimadores de riqueza reflejaron la existencia de una diversidad de diplópodos que aún no ha sido registrada completamente.

Palabras clave: riqueza, diversidad, comparación, similitud, milípedos, Parque Nacional Valle Nuevo.

Title: Comparative study of millipedes communities (Arthropoda: Diplopoda) present in highland savanna, pine forest, and broadleaf forest of Valle Nuevo National Park, Dominican Republic.

\section{ABSTRACT}

Diversity of Diplopoda (millipedes) communities in three types of vegetation in Valle Nuevo National Park (pine forest, highland savanna and broadleaf forest) during rainy season was compared. Three study stations corresponding to the three types of vegetation were established. The total richness recorded was 15 species. The broadleaf forest was the type of vegetation that recorded the highest value in species richness (12), while highland savanna presented the lowest value with eight species. The greatest abundance of specimens was recorded for pine forest (205), while the lowest for the broadleaf forest with 73 . The most abundant species in the study were Achromoporus platyurus and Prostemmiulus setosus. The community of broadleaf 
forest was the most diverse according to the value obtained with Shannon equity index and reciprocal of Simpson dominance index $\left(\mathrm{H}^{\prime}=2.25 ; \mathrm{D}=8.91\right)$. Highland savanna was the poorest in relation to the alpha diversity according to the results of these indices $\left(\mathrm{H}^{\prime}=1.42 ; \mathrm{D}=3.32\right)$. The similarity Index of Jaccard showed the lowest value to relate the highland savanna ecosystems and broadleaf forest (50\%), while the highest similarity was observed when comparing the highland savanna with the pine forest $(72 \%)$. This last result was statistically significant for a probability (p) of 0.05 showing that there is a high level of faunal similarity between both types of vegetation. This high similarity could be due to the structural homogeneity of the two types of vegetation for diplopods. A dendrogram that reflects these same results was made. The species accumulation curve reflected the existence of a variety of diplopods not yet registered.

Keywords: richness, diversity, comparison, similarity, Diplopoda, Valle Nuevo National Park.

\section{INTRODUCCIÓN}

El Parque Nacional Valle Nuevo comprende ecosistemas de gran valor, siendo su estudio de mucho interés al presentar condiciones climáticas y fisiográficas únicas en el país y en las Antillas. Este parque consta de cinco tipos de vegetación que varían dependiendo de la localidad: pinares, bosques latifoliados, manaclares (Guerrero et al., 2002; Núñez et al., 2006), así como sabanas de pajón y bosque ribereño (Peguero, 2013). El estudio de la fauna asociada a estos ecosistemas resulta de mucha importancia, tanto en el aspecto de los inventarios como de su dinámica ecológica. En este contexto los milpiés podrían considerarse uno de los grupos que mejor se prestan a tales fines por la importante función que desempeñan en la regulación de los procesos del suelo, siendo considerados ingenieros de este ecosistema junto con otros artrópodos (Bueno-Villegas, 2012). En la Hispaniola se han realizado múltiples estudios en el campo taxonómico, resultando en la descripción de 179 especies de diplópodos, distribuidas en 62 géneros, 18 familias y ocho órdenes (Suriel y Rodríguez, 2014), sin embargo, son muy pocos los estudios publicados que contemplan aspectos de la dinámica ecológica de los milpiés de la isla. El Parque Nacional Valle Nuevo cuenta con una evaluación ecológica integrada (Núñez, 2002), un plan de manejo (SEMARENA, 2005) y un plan de conservación (Núñez et al., 2006), sin embargo, en los estudios referidos no se incluye la fauna de artrópodos asociados al suelo.

Este estudio analiza las comunidades de diplópodos en tres tipos de vegetación del Parque Nacional Valle Nuevo, contribuyendo así al conocimiento en cuanto a la composición, diversidad de las comunidades y abundancia relativa del grupo en esta área protegida.

\section{OBJETIVO}

- Caracterizar y comparar las comunidades de milpiés en tres tipos de ambientes del Parque Nacional Valle Nuevo: sabana de pajón, pinar y bosque latifoliado.

\section{MATERIALES Y MÉTODOS}

\section{Tiempo del estudio y localidades de muestreo}

El presente trabajo se realizó entre junio-agosto del año 2010 y octubre del 2011, correspondiendo a los meses de la temporada lluviosa. Las estaciones de muestreo se ubicaron en el bosque latifoliado (332244 E; $2068070 \mathrm{~N})$, el pinar (331244 E; $2069220 \mathrm{~N})$ y en la sabana de pajón (331501 E; 2070693 N) comprendidos entre Las Espinas, en la entrada sur del parque y Sabana Quéliz, en dirección a la entrada norte. 
Diseño de muestreo y trabajo de campo. Se estableció una estación de estudio en cada uno de los tres tipos de vegetación considerados. En cada estación se delimitaron dos parcelas de muestreo, cada una con las dimensiones de 20 × 20 metros. Cada parcela de muestreo se dividió en cinco cuadrículas de 2 x 2 metros, distribuidas una en cada vértice del cuadrado y otra en el centro; este procedimiento sigue a Bueno-Villegas y Rojas Fernández (1999) y a Sánchez-Ruiz et al. (2009), con modificaciones referentes al esfuerzo de muestreo habiendo sido aumentados el tiempo de muestreo y el tamaño de las cuadrículas. Los diplópodos se colectaron manualmente por cinco personas de manera simultánea en cada cuadrícula durante 21 minutos. Los especímenes se conservaron vivos hasta la llegada al campamento de trabajo. Una vez allí, se colocaron en una solución de alcohol etílico al 12\% durante una o dos horas, con el fin de sacrificarlos garantizando un adecuado relajamiento muscular. Luego, se colocaron en envoltorios dentro de una solución de alcohol etílico al 70\%. Este procedimiento de manejo de los especímenes sigue a Pérez-Asso (1995). Las parcelas estudiadas de los tipos de vegetación pinar y sabana de pajón se encuentran frente al monumento La Pirámide, al otro lado de la carretera; la correspondiente al bosque latifoliado en la localidad mejor conocida como La Neverita. Se tomaron muestras de suelo para determinar el porcentaje de materia orgánica de cada tipo de vegetación y relacionarlo con la abundancia de diplópodos. Estas muestras fueron analizadas en un laboratorio de referencia privado.

Los especímenes colectados se identificaron hasta el táxon más bajo posible utilizando las claves y descripciones correspondientes (Loomis, 1941; Pérez-Asso, 2009) siendo depositados en la colección de diplópodos del Museo Nacional de Historia Natural "Prof. Eugenio de Jesús Marcano" (MNHNSD). Una vez realizadas las determinaciones taxonómicas, se organizaron los datos en Tablas, a los fines de facilitar su análisis. Para la nomenclatura taxonómica mayor se siguió a Hoffman (1979).

Análisis estadístico. Para obtener una mejor aproximación en el conocimiento de las comunidades estudiadas, se consideraron varios métodos para abordar los dos componentes principales de la diversidad: riqueza de especies y uniformidad (Magurran, 2004). Basados solamente en la presencia-ausencia de las especies se utilizaron riqueza de especies (S) y los estimadores no paramétricos Chao 2, Jackknife de primer orden y Bootstrap; mientras que tomando en consideración la abundancia proporcional (A), se determinaron: índice de diversidad de Shannon $\left(H^{\prime}\right)$, índice de dominancia de Simpson $(\lambda)$ y el estimador no paramétrico Chao 1 , siguiendo a Moreno (2001). Mediante el coeficiente de Jaccard (J) se determinó la similitud entre las zonas de estudio y para determinar si los valores de similitud obtenidos fueron estadísticamente significativos se utilizó la tabla de significancia de Real (1999). Para la realización de los análisis estadísticos se utilizaron los programas Infostat 2014, EstimateS 9.10 y Statistica 12.

\section{RESULTADOS Y DISCUSIÓN}

Riqueza y abundancia. La fauna de diplópodos presente en las áreas de estudio estuvo representada por 15 especies, equivalente a $8.3 \%$ de las especies registradas para La Hipaniola. Estas especies se distribuyen en seis familias y nueve géneros. El total de ejemplares colectados fue de 475 (Tabla I). El 100\% de las especies registradas son endémicas de la isla (Fig. 1, A-I). Las especies Achromoporus occultus y Prostemmiulus setosus, estuvieron presentes en las seis parcelas de los tres sitios de muestreo, mostrando la más amplia distribución. Las especies más abundantes fueron Achromoporus platyurus (127) y Prostemmiulus setosus (105). Las especies menos abundantes fueron Henicomus septiporus y Docodesmus sp. con un ejemplar de cada especie. La familia con mayor riqueza de especies fue Chelodesmidae en las tres áreas de estudio, coincidiendo este resultado con el obtenido por Sánchez Ruiz et al. (2009) en un pinar del Parque Nacional José del Carmen Ramírez. 
La riqueza de especies presentó resultados similares en el pinar y el bosque latifoliado, 11 y 12 especies, respectivamente. La sabana de pajón, en cambio, presentó el menor número de especies encontradas en todo el estudio, con apenas 8 de las 15 registradas. El bosque latifoliado además de ser el más rico fue el ecosistema que presentó mayor número de especies exclusivas: Fuhrmannodesmidae Morfo B, Henicomus septiporus, Spirobolellus sp. y Prostemmiulus gracilipes, estos resultados podrían indicar que la heterogeneidad de especies de plantas que suele encontrarse en este tipo de vegetación favorece a la riqueza de diplópodos.

A diferencia de los resultados de riqueza de especies, la abundancia registró su más bajo valor en el bosque latifoliado, con apenas 73 ejemplares en todo el muestreo. El valor más alto de abundancia se presentó en el pinar, 205 ejemplares, siguiéndole la sabana de pajón con 197 (Tabla I). Probablemente esto se deba a la abundancia de recursos utilizados por los diplópodos. En el caso del pinar, los microhábitats producidos por troncos desprendidos y en estado de descomposición de Pinus occidentalis pudieran estar actuando como un factor favorecedor. Para la sabana de pajón la abundante materia orgánica (17\%) explicables en la naturaleza del material vegetal proveniente de la Danthonia domingensis, especie dominante en esta vegetación. Esta especie, al igual que otras de la familia Poacea, es de simple descomposición y de ciclo corto. Además de $D$. domingensis, la sabana de pajón presenta otras herbáceas, con un solo evento reproductivo durante su ciclo de vida, muriendo después de fructificar (Peguero, 2013); además, las plantas con sistemas radiculares densos, así como raíces finas y abundantes como las poaceas proporcionan más materia orgánica que las masas forestales (Porta et al., 2013). Esto, sumado a los microambientes creados por las bases de los pajones, los que según nuestras observaciones son preferidos por una gran cantidad de diplópodos, pudiera explicar la mayor abundancia obtenida en este ecosistema. Por otro lado, el bosque latifoliado presenta el más bajo porcentaje de materia orgánica $(2.3 \%)$ debido probablemente a una menor producción de hojarasca relacionada con un ciclo de vida más largo y un sistema radicular menos denso de las plantas de este ecosistema en relación con pajones, así como una pendiente pronunciada en las áreas de muestreo. El porcentaje de materia orgánica en el pinar fue de 8\%.

La mayor abundancia de diplópodos encontrada en el pinar contrasta con los resultados de Sánchez-Ruiz et al. (2009), siendo necesario considerar el mayor esfuerzo de muestreo realizado en la presente investigación. En el total del muestreo para las tres áreas, la familia más abundante fue Chelodesmidae (57.89\%) esa supremacía también se pudo observar en los resultados parciales donde obtuvo mayores abundancias en dos de los tres hábitats muestreados siendo mínimamente superada solo en el bosque latifoliado por la familia Stemmiulidae por apenas dos individuos. Estos resultados en abundancia, en combinación con los obtenidos en riqueza de especies, destacan a esta familia como la más diversa y abundante. Es probable que tanto la abundancia como la riqueza de Chelodesmidae en las tres áreas estudiadas se relacionen con su plasticidad trófica. Este es considerado un importante factor regulador de poblaciones de milpiés (Loranger-Merciris et al., 2008), no obstante se ha documentado la tendencia de los milpiés para alimentarse preferencialmente de cierto tipo de hojarasca (Warren y Zou, 2002; Loranger-Merciris et al., 2008) por lo tanto esta familia podría ser la más exitosa en aprovechar los recursos alimentarios disponibles en las áreas muestreadas. La coincidencia en altos valores de abundancia entre el pinar y la sabana de pajón podría estar relacionada con la estrecha asociación entre ambos ecosistemas (Peguero, 2013). Este resultado se debe fundamentalmente a los quélodesmidos, los cuales, a diferencia de los restantes grupos, tienen apreciable movilidad y podrían estar desplazándose entre estos dos ecosistemas. Esta familia representó el 49.2\% del total de los diplópodos en el pinar y el $66.4 \%$ en la sabana de pajón. 

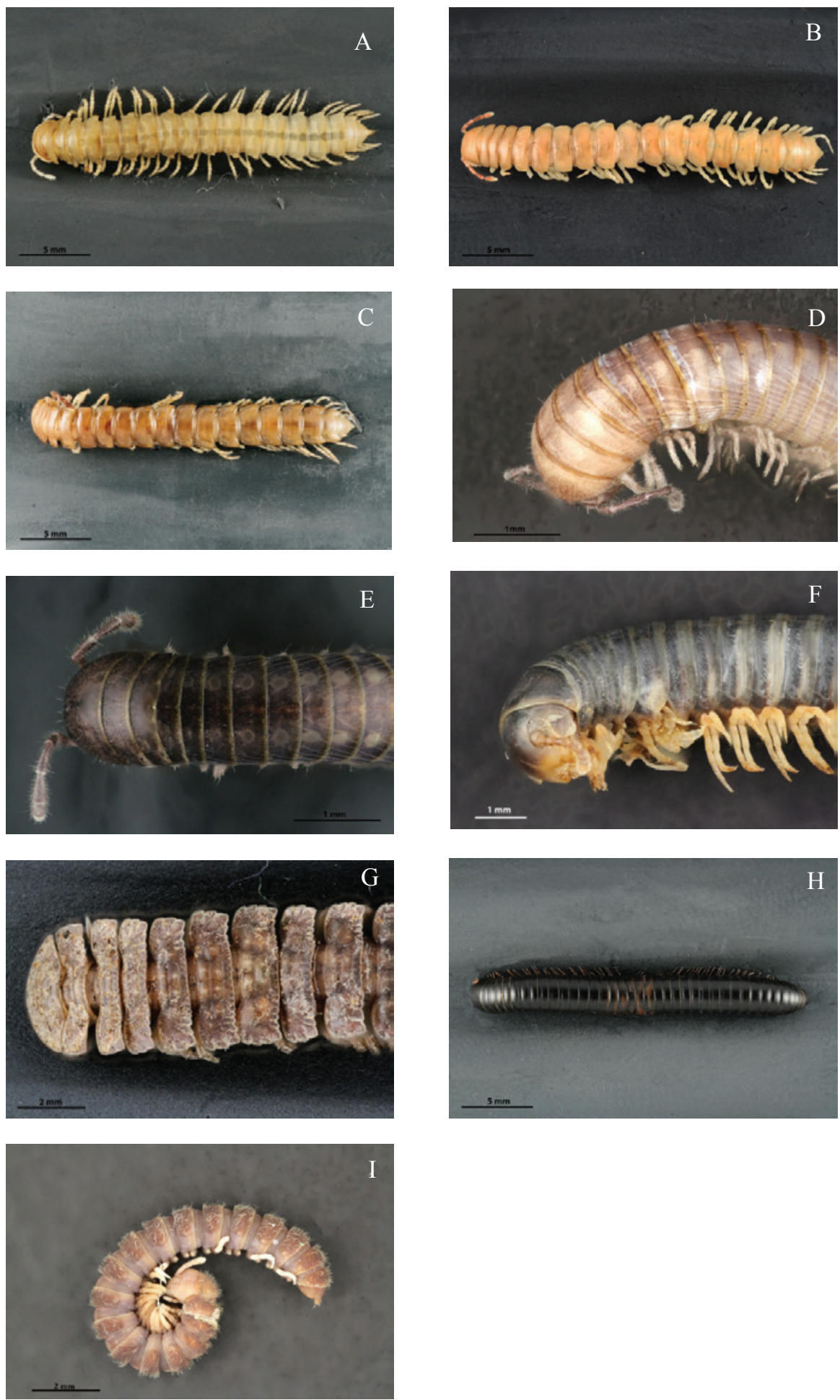

Figura 1, A-I. Fotos de algunas de las especies de milpiés encontradas en Valle Nuevo. A, Achromoporus andujari. B, Achromoporus vallenuevo. C, Achromoporus platyurus. D, Prostemmiulus scaurus. E, Prostemmiulus setosus. F, Prostemmiulus gracilipes. G, Docodesmus angustus. H, Spirobolellus sp. I, Fuhrmannodesmidae Morfo B. 
Tabla I. Abundancia absoluta por especie de diplópodos del Parque Nacional Valle Nuevo en las seis parcelas de las tres áreas muestreadas.

\begin{tabular}{|c|c|c|c|c|c|c|c|c|c|}
\hline \multirow{2}{*}{ Familia } & \multirow{2}{*}{ Especie } & \multirow{2}{*}{ Abreviaturas } & \multicolumn{2}{|c|}{ Pinar } & \multicolumn{2}{|c|}{$\begin{array}{c}\text { Bosque } \\
\text { latifoliado }\end{array}$} & \multicolumn{2}{|c|}{$\begin{array}{l}\text { Sabana de } \\
\text { pajón }\end{array}$} & \multirow{2}{*}{$\begin{array}{l}\text { Total por } \\
\text { especie }\end{array}$} \\
\hline & & & $\mathrm{PN}-1$ & $\mathrm{PN}-2$ & BL-1 & BL-2 & SP-1 & SP-2 & \\
\hline \multirow{5}{*}{ Chelodesmidae } & Achromoporus andujari & Aan & 13 & 7 & 7 & 0 & 6 & 12 & 45 \\
\hline & Achromoporus concolor & Aco & 1 & 2 & 0 & 0 & 0 & 0 & 3 \\
\hline & Achromoporus occultus & Aoc & 32 & 20 & 7 & 2 & 4 & 11 & 76 \\
\hline & Achromoporus platyurus & Apl & 28 & 10 & 3 & 0 & 29 & 57 & 127 \\
\hline & Achromoporus vallenuеvo & Ava & 6 & 4 & 2 & 0 & 12 & 0 & 24 \\
\hline \multirow{2}{*}{ Fuhrmannodesmidae } & Morfo $A$ & FMA & 2 & 0 & 1 & 5 & 1 & 0 & 9 \\
\hline & Morfo $B$ & FMB & 0 & 0 & 2 & 0 & 0 & 0 & 2 \\
\hline \multirow{3}{*}{ Pyrgodesmidae } & Docodesmus angustus & Dan & 1 & 3 & 4 & 0 & 3 & 0 & 11 \\
\hline & Docodesmus sp. & Dsp & 1 & 0 & 0 & 0 & 0 & 0 & 1 \\
\hline & Henicomus septiporus & Hse & 0 & 0 & 0 & 1 & 0 & 0 & 1 \\
\hline Siphonophoridae & Siphonophora platops & Spl & 1 & 3 & 0 & 0 & 1 & 0 & 5 \\
\hline Spirobolellidae & Spirobolellus sp. & Ssp & 0 & 0 & 11 & 4 & 0 & 0 & 15 \\
\hline \multirow{3}{*}{ Stemmiulidae } & Prostemmiulus gracilipes & Pgr & 0 & 0 & 9 & 1 & 0 & 0 & 10 \\
\hline & Prostemmiulus scaurus & Psc & 12 & 27 & 2 & 0 & 0 & 0 & 41 \\
\hline & Prostemmiulus setosus & Pse & 21 & 11 & 7 & 5 & 59 & 2 & 105 \\
\hline Total por localidad & & & \multicolumn{2}{|c|}{205} & \multicolumn{2}{|c|}{73} & \multicolumn{2}{|c|}{197} & - \\
\hline Total general & & & & & & & & & 475 \\
\hline
\end{tabular}

Parcelas 1 y 2 en pinar $=$ PN-1 y PN-2. Parcelas 1 y 2 en bosque latifoliado = BL-1 y BL-2. Parcelas 1 y 2 en sabana de pajón $=$ SP-1 y SP-2.

Diversidad. Los resultados obtenidos con el índice de diversidad de Shannon para todo el muestreo realizado (las seis parcelas muestreadas en los tres tipos de vegetación), permiten asumir una diversidad media de diplópodos (2.05), ver Tabla II. De igual manera, los resultados obtenidos con el recíproco del índice de Simpson arrojaron un valor de diversidad media (5.95). Esto probablemente se deba a un buen estado de conservación de la zona.

En cuanto a la diversidad por tipo de hábitat, el bosque latifoliado resultó ser el de mayor equidad, con un valor de 2.25 para el índice de Shannon. Este resultado explica que la proporción relativa de cada una de las especies presentes es más homogénea en el caso del bosque latifoliado comparado con el pinar y la sabana de pajón, cuyos valores de equidad fueron menores, $1.96 \mathrm{y}$ 1.42 , respectivamente.

El bosque latifoliado fue el ecosistema que presentó el valor más bajo de dominancia de especie (0.12) al aplicar el índice de Simpson, siendo su recíproco 8.91, resultados que se corresponden con los obtenidos del índice de Shannon (mayor diversidad se corresponde con más baja dominancia). Este resultado probablemente se corresponda con la existencia de una mayor diversidad de hábitats en el bosque latifoliado debido a la propia estructura de esta vegetación. La sabana de pajón y el pinar resultaron con valores más altos de dominancia, 0.3 y 0.17 , respectivamente. La distribución equitativa en estos dos ecosistemas fue menor en comparación con el bosque laifoliado, siendo el recíproco del índice de Simpson 5.95 y 3.92 
respectivamente, reflejando una menor diversidad de especies; tanto la sabana de pajón como el pinar son ecosistemas bastante homogéneos en cuanto a hábitats disponibles si los comparamos con el bosque latifoliado.

Tabla II. Parámetros ecológicos de los diplópodos del Parque Nacional Valle Nuevo por tipos de vegetación.

\begin{tabular}{cccc}
\hline & Pinar & Bosque latifoliado & Sabana de pajón \\
\hline Riqueza (S) & 11 & 12 & 8 \\
Abundancia (A) & 205 & 73 & 197 \\
Shannon (H') & 1.92 & 2.25 & 1.42 \\
Simpson ( $\lambda$ ) & 0.17 & 0.12 & 0.3 \\
Recíproco de & 5.95 & 8.91 & 3.32 \\
Simpson (D) & & & \\
\hline
\end{tabular}

Curva de acumulación de especies y estimadores no paramétricos. Como se muestra en las figuras 2, 3 y 4, el número de especies registrado se incrementa conforme se intensifican los muestreos, sin conseguir una curva asintótica, es decir, las curvas de especies observadas no manifiestan un punto de inflexión. Estos resultados muestran que el inventario no fue completo con base en la riqueza estimada y se espera que se sigan agregando especies conforme se aumente el esfuerzo de muestreo. No obstante, en todos los casos la eficiencia de muestreo sobrepasó el $75 \%$, considerada una proporción de especies relativamente alta, tomando en consideraron el reducido tamaño de la muestra (Jiménez-Valverde y Hortal, 2003).

El algoritmo de Jackknife 1 estimó el mayor número de especies en el ensamble para pinar y sabana de pajón, 13 y 10 respectivamente (Tabla III), lo que significa, un menor valor de representatividad del muestreo en estas áreas; mientras que Bootstrap estimó la mayor riqueza de especies en bosque latifoliado, 16 especies. Por otro lado, Chao 1 y Chao 2 estimaron el menor número de especies en los tres ecosistemas, acercándose siempre al valor de riqueza encontrada en el campo y correspondiendo con una representatividad del muestreo más alta. Dado que las curvas de los estimadores de riqueza, al igual que la curva de especies observadas, no alcanzan una estabilidad, se requiere un mayor esfuerzo de muestreo para que los estimadores den una aproximación más significativa de la riqueza de especies en las áreas muestreadas.

Tabla III. Valores de los estimadores de riqueza.

\begin{tabular}{lccc}
\hline & Pinar & Bosque latifoliado & Sabana de pajón \\
\hline Sobs & 11 & 12 & 8 \\
Chao 1 & 12 & 12 & 9 \\
Chao 2 & 12 & 15 & 9 \\
Jackknife 1 & 13 & 15 & 10 \\
Boostrap & 12 & 16 & 9 \\
\hline
\end{tabular}




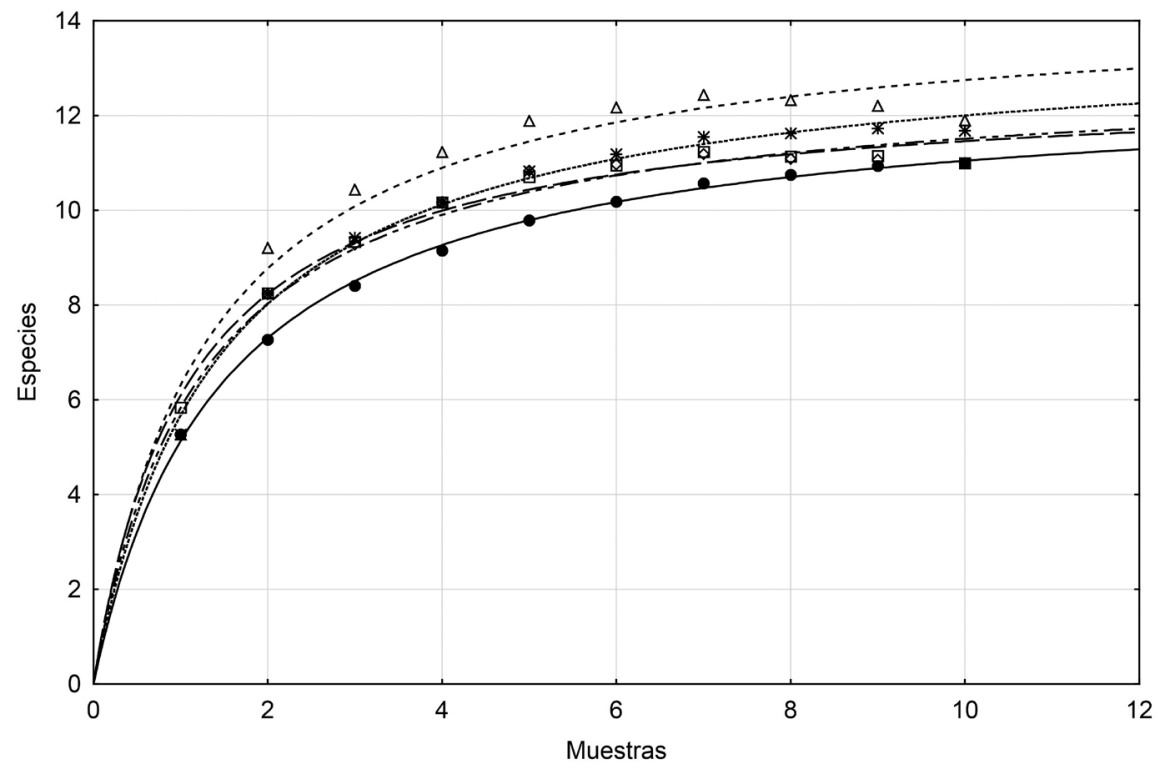

- Sobs a Chao1 $\diamond$ Chao2 $\Delta$ Jack1 * Boostrap

Figura 2. Curva de acumulación de especies y estimadores no paramétricos en el pinar.

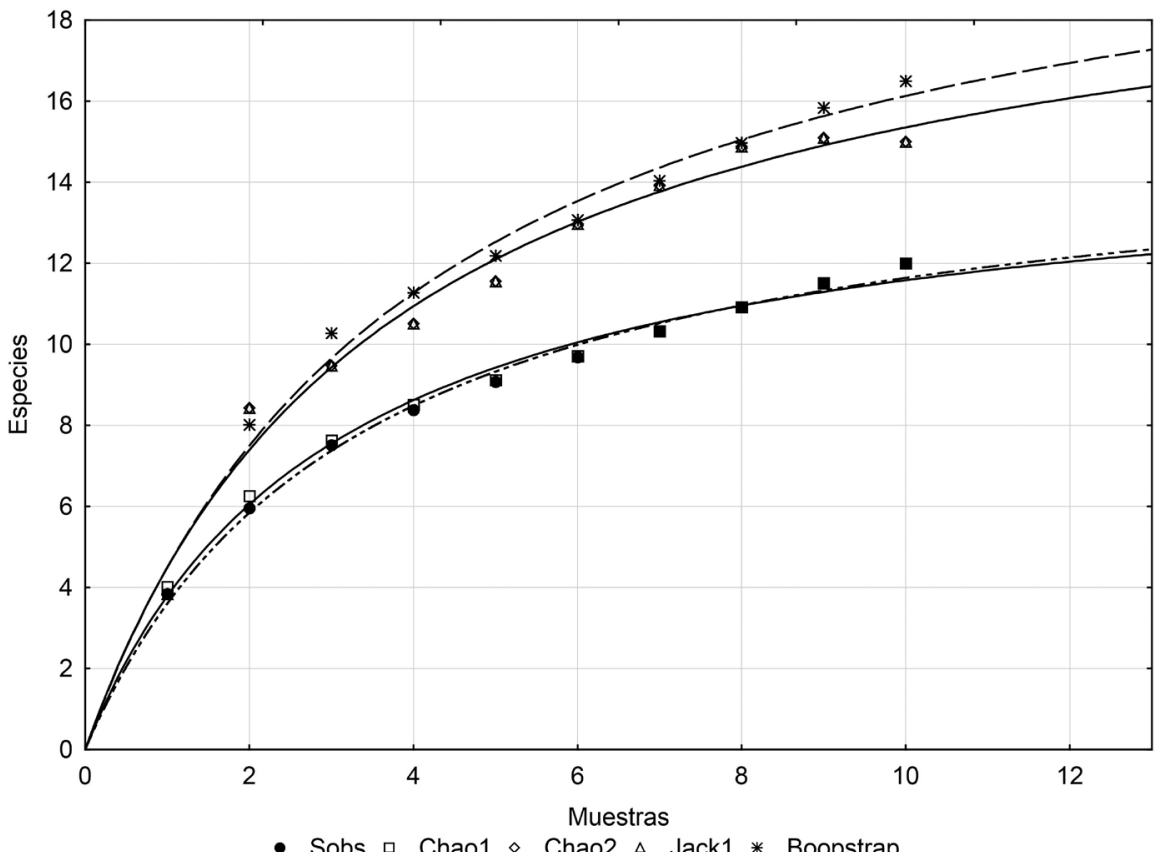

- Sobs a Chao1 $\diamond$ Chao2 $\Delta$ Jack1 * Boopstrap 


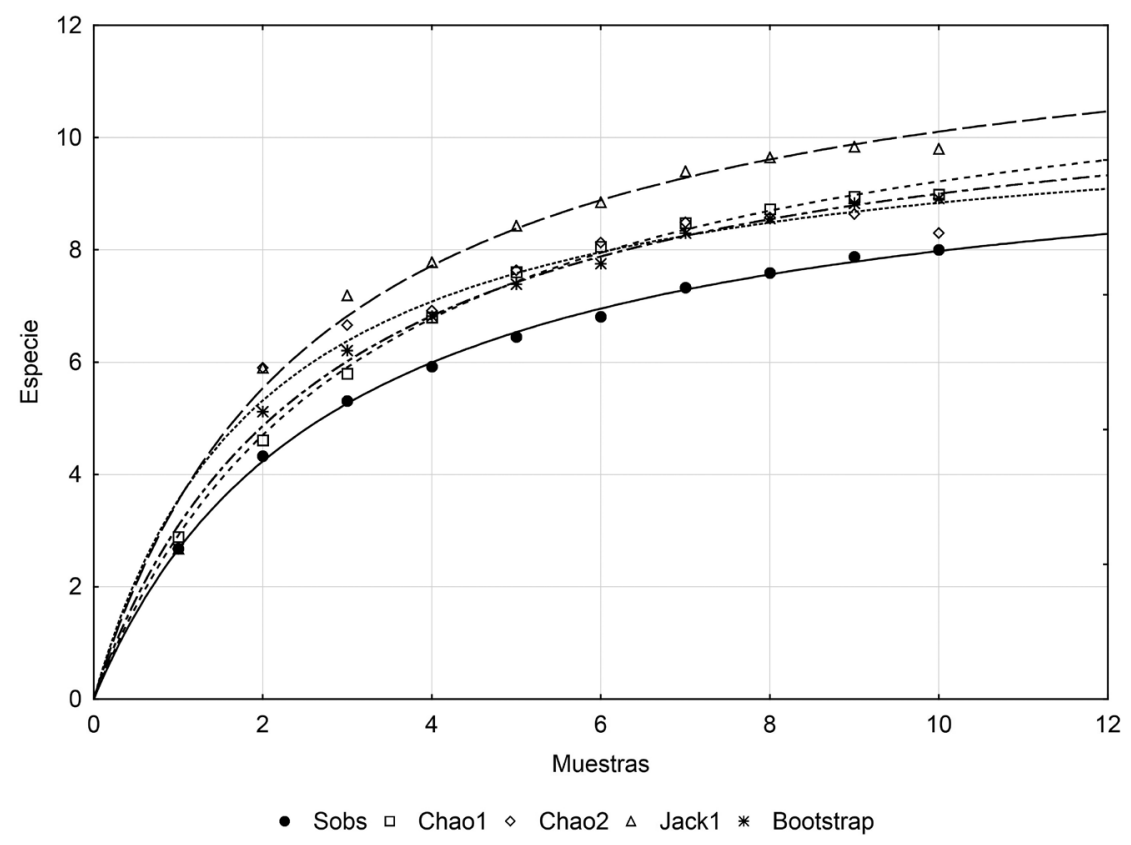

Figura 4. Curva de acumulación de especies y estimadores no paramétricos en la sabana de pajón.

Similitud. Las similitudes pareadas entre tipos de vegetación, registradas con el índice de Jaccard, resultaron con valores que podrían ser considerados elevados (50\% o más); sin embargo, en ningún caso la similitud entre las áreas alcanzó 80\%. El valor más bajo de similitud (50\%) se obtuvo al comparar la sabana de pajón y el bosque latifoliado, ecosistemas que compartieron siete especies (Tabla IV). Este resultado puede deberse a diferencias en la estructura vegetal de ambos ecosistemas, debiendo considerarse también que estas locaciones se encuentran bastante apartadas entre sí (Peguero, 2013). Este resultado fue estadísticamente no significativo para una probabilidad (p) de 0.05. La mayor similitud (72\%), compartiendo ocho especies, se obtuvo al comparar la sabana de pajón con el pinar. Estos resultados muestran que existe un alto grado de semejanza faunística entre ambos tipos de vegetación. Esta alta similitud podría deberse a la homogeneidad estructural de estos dos tipos de vegetación para los diplópodos, los cuales no estarían restringidos por la estructura arbórea en este caso particular. El valor obtenido entre estos dos ecosistemas resulta significativo para una probabilidad (p) de 0.05 de acuerdo a la tabla de significancia (Real, 1999), habiendo 11 especies entre sabana de pajón y pinar $(\mathrm{N}=11)$ y siendo el valor esperado 0.63 (63\%). La similitud entre el bosque latifoliado y el pinar fue de $60 \%$, compartiendo ocho especies. Este valor fue significativo para una probabilidad (p) de 0.05 , siendo el valor esperado en la Tabla de significancia de 0.60 (Real, 1999). Estos porcentajes son explicados con el dendrograma realizado a partir de la matriz de similitud obtenida con el índice de Jaccard (Fig. 5), estando agrupados pinar y sabana de pajón por poseer mayor similitud.

Tabla IV. Matriz de similitud generada con el índice de Jaccard.

\begin{tabular}{|c|c|c|c|}
\hline & Pinar & Bosque latifoliado & Sabana de pajón \\
\hline Pinar & 1 & & \\
\hline Bosque latifoliado & 0.60 & 1 & \\
\hline Sabana de pajón & 0.72 & 0.50 & 1 \\
\hline
\end{tabular}




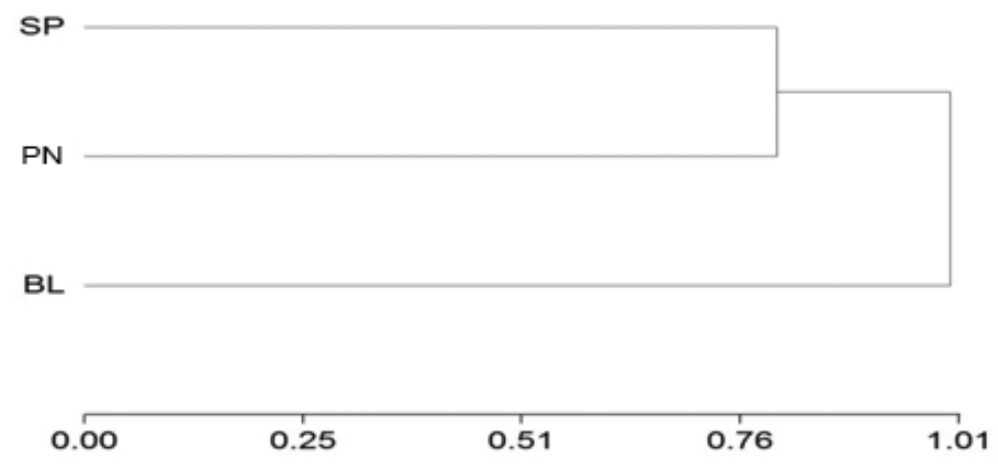

Figura 5. Dendrograma. $\mathrm{SP}=$ Sabana de pajón. $\mathrm{PN}=$ Pinar. $\mathrm{BL}=$ Bosque latifoliado.

\section{RECOMENDACIONES}

- Ampliar el estudio en el Parque Nacional Valle Nuevo incluyendo otros tipos de vegetación (manaclares y la vegetación ribereña).

- Tomar en cuenta la temporada seca e incluir nuevos métodos de colecta con el fin de hacer comparaciones entre temporadas y ampliar la posibilidad de localizar las especies no registradas en este estudio.

- Tomar medidas de conservación para los ecosistemas presentes, con especial énfasis en la sabana de pajón, la que está visiblemente más alterada entre los ecosistemas estudiados.

\section{AGRADECIMIENTOS}

Al Museo Nacional de Historia Natural "Prof. Eugenio de Jesús Marcano" por el apoyo brindado, parte esencial de la realización de este trabajo de investigación. Al Fondo Nacional de Innovación y Desarrollo Científico y Tecnológico (FONDOCYT) del Ministerio de Educación Superior, Ciencia y Tecnología (MESCYT), de la República Dominicana por el auspicio del proyecto de investigación del que se desprende este trabajo. A Celeste Mir, América Sánchez, Cristian Marte, Gabriel de los Santos, Miguel Santiago Nuñez, Solanlly Carrero, Ángel Pimentel y Ruth Bastardo por la colaboración y apoyo brindado.

\section{LITERATURA CITADA}

Bueno-Villegas, J. 2012. Diplópodos: los desconocidos formadores de suelo. CONABIO. Biodiversitas, 102: 1-5.

Bueno-Villegas, J. y P. Rojas Fernández. 1999. Fauna de milpiés (Arthropoda: Diplopoda) edáficos de una selva de los Tuxtla. Acta zoológica mexicana, 76: 59-83.

Guerrero, A., N. Ramírez, A. Veloz y B. Peguero. 2002. Vegetación y flora del Parque Nacional Juan Bautista Pérez Rancier (Valle Nuevo). 34-56. En: Evaluación Ecológica Integrada Parque Nacional Juan Bautista Pérez Rancier. 147 pp.

Hoffman, R. L. 1979. Clasification of the Diplopoda. Mem. Mus. His. Nat. Geneve. 209 pp.

Jiménez-Valverde A. y J. Hortal. 2003. Las curvas de acumulación de especies y la necesidad de evaluar la calidad de los inventarios biológicos. Revista Ibérica de Aracnología, 8:151-161. 
Loomis, H. F. 1941. Millipeds collected in Puerto Rico and the Dominican Republic by Dr. P. J. Darlington in 1938. Bulletin of the Museum of Comparative Zoology at Harvard College, 88: $17-80$.

Loranger-Merciris, G., D. Imbert, F. Bernhard Reversat, P. Lavelle, J. F. Ponge. 2008. Litter N-content influences soil millipede abundance, species richness and feeding preferences in a semi-evergreen dry forest of Guadeloupe (Lesser Antilles), Biology and Fertility of Soils, (45)1:93-98.

Magurran, A. E. 2004. Measuring biological diversity, Blackwell Publishing: Oxford, UK. $256 \mathrm{pp}$.

Moreno, C. E. 2001. Métodos para medir la biodiversidad. Manuales y Tesis, Sociedad Entomológica Aragonesa (SEA), Vol. 1. Zaragosa, 84 pp.

Núñez, F. 2002. Evaluación ecológica integrada Parque Nacional Juan Bautista Pérez Rancier (Valle Nuevo). Secretaría de Estado de Medio Ambiente y Recursos Naturales/ Fundación Moscoso Puello. 147 pp.

Núñez, F., N. Ramírez, M. McPherson y F. Portoreal. 2006. Plan de conservación Parque Nacional Juan Bautista Pérez Racier (Valle Nuevo). Editora Amigo del Hogar. Santo Domingo, República Dominicanam. 87 pp.

Peguero, B. 2013. Diversidad y Estructura de la Vegetación en la Sabana de Pajón de Valle Nuevo. Moscosoa, 18: 137-153.

Pérez-Asso, A. R. 1995. Colecta y conservación de diplópodos. Cocuyo, 2: 2-3.

Pérez-Asso, A. R. 2009. El género Achromoporus (Diplopoda: Polydesmida: Chelodesmidae) en República Dominicana: especies nuevas y sinonimias. Solenodon, 8: 33-81.

Porta J., M. López-Acebedo y R. M. Poch. 2013. Edafología: uso y protección de suelos. MundiPresnsa. 608pp.

Real, R. 1999. Tables of significant values of Jaccard's index of similarity. Miscellania Zoologica, 22(1): 29-40.

Sánchez-Ruiz, A., C. Suriel y G. de los Santos. 2009. Muestreo postfuego de artrópodos de suelo en bosque de pinos del Parque Nacional José del Carmen Ramírez, República Dominicana. Novitates Caribaea, 2: 30-39.

Secretaría de Estado de Medio Ambiente y Recursos Naturales (SEMARENA). Abril 2005. Resumen Ejecutivo del Plan de Manejo del Parque Nacional Juan Bautista Pérez Rancier (Valle Nuevo) 2007 - 2011. Santo Domingo, República Dominicana. 82 pp.

Suriel, C. y K. Rodríguez. 2014. Inventario de los milípedos (Clase Diplopoda) de La Hispaniola: actualización 2008-2013. En: VIII Congreso de la Biodiversidad Caribeña, del 29 de enero al 1 de febrero 2014; Universidad Autónoma de Santo Domingo, República Dominicana.

Warren, M.W. y X. Zou. 2002. Soil macrofauna and litter nutrients in three tropical tree plantations on a disturbed site in Puerto Rico. Forest Ecology and Management, 170:161-171.

[Recibido: 17 de agosto, 2015. Aceptado para publicación: 15 de septiembre, 2015] 